\title{
Neuritis óptica inflamatoria
}

\section{Inflammatory optic neuritis}

\author{
T. Ayuso' ${ }^{1}$ D. Aliseda ${ }^{2}$, I. Ajuria ${ }^{1}$, B. Zandío ${ }^{1}$, S. Mayor ${ }^{1}$, M.C. Navarro ${ }^{1}$
}

\section{RESUMEN}

La neuritis óptica inflamatoria (NO) es la causa más frecuente de pérdida visual aguda en adultos jóvenes. Aunque el pronóstico visual es excelente en la mayoría de los casos, muchos pacientes desarrollarán otra patología como esclerosis múltiple en la evolución posterior.

La historia natural de la NO ha sido estudiada en múltiples trabajos en los últimos años; uno de los más importantes es el Optic Neuritis Treatment Trial.

La Resonancia Magnética tiene un papel fundamental en el diagnóstico etiológico de la NO y en la predicción del riesgo de conversión a esclerosis múltiple.

Recientemente se han incorporado nuevas técnicas exploratorias como la tomografia de coherencia óptica, útil para el diagnóstico y pronóstico; se han identificado biomarcadores séricos que ayudan en el diagnóstico de otras patologías de naturaleza autoinmune que producen $\mathrm{NO}$.

Un mejor conocimiento de los datos clínicos y exploratorios de la NO típica permitirá un estudio diagnóstico más rápido y certero. El tratamiento de la NO con esteroides debe ser individualizado teniendo en cuenta que no modifican el pronóstico a largo plazo y en pacientes con alto riesgo de conversión a esclerosis múltiple debe plantearse terapia inmunomoduladora.

Este trabajo revisa los datos existentes en la literatura referentes a las manifestaciones clínicas, el diagnóstico etiológico y diferencial y tratamiento de la NO inflamatoria.

Palabras clave. Neuritis óptica inflamatoria. Esclerosis múltiple. Síndrome clínico aislado. Neuritis óptica bilateral.

\section{ABSTRACT}

Inflammatory Optic Neuritis (ON) is the most frequent cause of acute visual loss in young adults. Although the visual prognosis is excellent in the majority of cases, many patients develop pathology, such as multiple sclerosis, in its subsequent evolution.

The natural history of $\mathrm{ON}$ has been studied in numerous works in recent years; one of the most important of which is Optic Neuritis Treatment Trial.

Magnetic Resonance plays a fundamental role in the etiological diagnosis of $\mathrm{ON}$ and in predicting the risk of conversion into multiple sclerosis.

New exploratory techniques have recently been incorporated, such as optical coherence tomography, useful for diagnosis and prognosis; serum biomarkers have been identified in the diagnosis of other pathologies with an autoimmune nature that produce $\mathrm{ON}$.

A better understanding of the clinical and exploratory data of typical ON will make a more rapid and accurate diagnostic study possible. Treatment of ON with steroids must be individualised bearing in mind that they do not alter the long-term prognosis and an immunomodulating therapy must be proposed for patients with a high risk of conversion into multiple sclerosis.

This article reviews the existing data in the literature on its clinical manifestations, its etiological and differential diagnosis, and the treatment of inflammatory ON.

Key words. Inflammatory optic neuritis. Multiple sclerosis. Isolated clinical syndrome. Bilateral optic neuritis.
1. Servicio de Neurología. Hospital de Navarra. Pamplona

2. Servicio de Oftalmología. Hospital de Navarra. Pamplona

Recepción: 23 de febrero de 2009

Aceptación provisional: 12 de mayo de 2009

Aceptación definitiva: 10 de junio de 2009

\author{
Correspondencia \\ Teresa Ayuso Blanco \\ Servicio de Neurología. Hospital de Navarra. \\ Irunlarrea, 3 \\ 31008 Pamplona \\ Tfno. 848422292 \\ Fax 848422303 \\ E-mail: tayusob@yahoo.es
}




\section{INTRODUCCIÓN}

La causa más frecuente de pérdida visual aguda en adultos jóvenes es la neuritis óptica (NO) idiopática cuya patogénesis es un proceso inflamatorio y desmielinizante del nervio óptico. La forma más habitual, en cuanto a localización de la inflamación, es la retrobulbar donde la apariencia del disco óptico es normal a diferencia de la papilitis en donde la inflamación es anterior. Son también variantes de la neuritis óptica, la neurorretinitis o inflamación intraocular del nervio óptico y de la retina peripapilar y perineuritis o afectación inflamatoria de las vainas del nervio óptico ${ }^{1}$.

Uno de los aspectos más interesantes de esta entidad es su relación con la esclerosis múltiple (EM) puesto que una alta proporción de pacientes con NO desarrollará EM en años posteriores. Hasta un 75\% de los casos cumplirán criterios de EM en los siguientes 15 años y un $20 \%$ de pacientes con EM debutan con NO como síndrome clínico aislado. Son muchos los estudios que han intentado aclarar cuáles son los factores de riesgo de conversión de $\mathrm{NO}$ a EM analizando neuroimagen, líquido cefalorraquídeo (LCR) y marcadores genéticos para identificar pacientes de alto riesgo que pueden beneficiarse de un tratamiento precoz ${ }^{2}$.

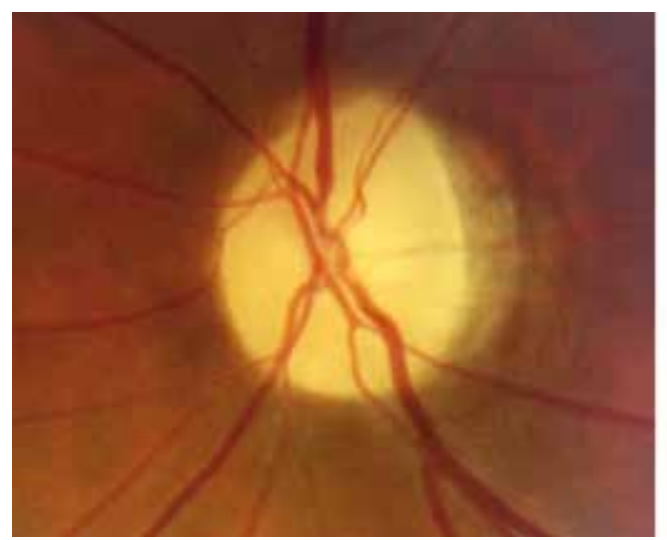

Otras patologías diferentes de la esclerosis múltiple pueden originar o simular neuritis óptica inflamatoria por lo que es necesario reconocer aquellas formas atípicas de NO en las que es preciso ampliar el estudio etiológico. En los últimos años se han realizado importantes avances en el conocimiento de alguna de ellas, también de naturaleza autoinmune e inflamatoria, que pueden ser identificadas y diferenciadas de la EM gracias a la determinación de biomarcadores específicos.

\section{MANIFESTACIONES CLÍNICAS Y EXPLORACIÓN}

La NO aparece en pacientes jóvenes, de los 14 a los 45 años y predomina en mujeres en una proporción 3:1. El síntoma clave es la disminución de agudeza visual que varía en intensidad desde leve a una ausencia de percepción de la luz. Su comienzo puede ser agudo o subagudo y existe una pérdida de visión cromática. Suele ser unilateral la mayoría de las veces. En el $90 \%$ de los casos se acompaña o precede de dolor con los movimientos oculares, atribuido al estiramiento de la vaina dural alrededor del nervio inflamado y los síntomas se exacerban con el calor (fenómeno de Uhthoff) y con el ejercicio físico ${ }^{2}$.

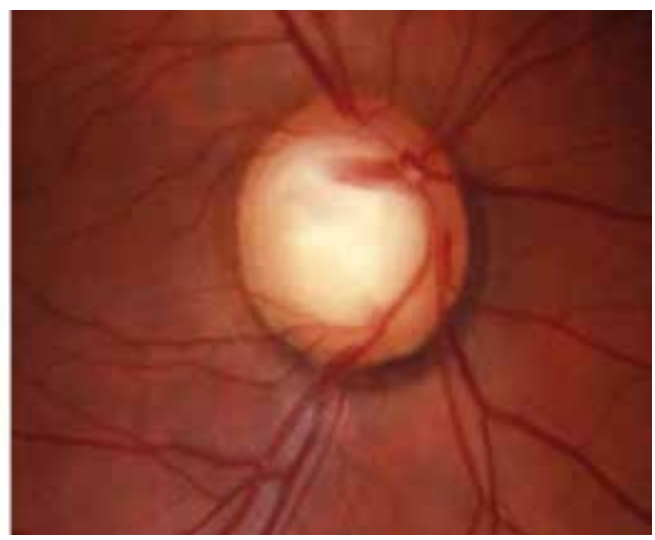

Figura 1. En la evolución en el tiempo, la neuritis puede dejar signos de atrofia óptica claramente diferenciables de la atrofia óptica glaucomatosa. A la izquierda atrofia óptica secundaria a neuritis óptica recidivante de ojo izquierdo. Obsérvese la pérdida de coloración difusa con vasos centrados, en este caso existe una pequeña atrofia coriorretiniana peripapilar en forma de semiluna temporal, secundaria a un defecto miópico asociado. A la derecha una excavación glaucomatosa en ojo derecho. En esta retinografia de $30^{\circ}$ centrales, la pérdida de coloración corresponde a la excavación central con conservación del ribete neural y desplazamiento de los vasos. 
El examen oftalmológico ha de ir dirigido a confirmar la sospecha de NO inflamatoria, en segundo lugar a esclarecer la causa para instaurar el tratamiento y, por último, a establecer el pronóstico visual y neurológico.

El hallazgo más frecuente en la exploración es el defecto pupilar aferente en el ojo afectado o fenómeno de Marcus Gunn salvo en casos de neuritis bilateral o disfunción visual residual si el paciente ha tenido un episodio previo en el ojo contralateral.

La exploración del fondo de ojo es normal en dos tercios de los pacientes exis- tiendo signos inflamatorios en los casos de papilitis. En general, no hay correlación entre el grado de inflamación aparente y el defecto visual o campimétrico. En las formas crónicas, se puede apreciar palidez o atrofia papilar y surcos retinianos si la NO es recurrente (Fig. 1).

Puede haber pérdida de campo visual difusa o focal en el ojo afecto en el $50 \%$ de los casos. Otros defectos campimétricos incluyen escotomas centrales o centrocecales, aumento de la mancha ciega, cuadrantanopsia o hemianopsia (Fig. 2).

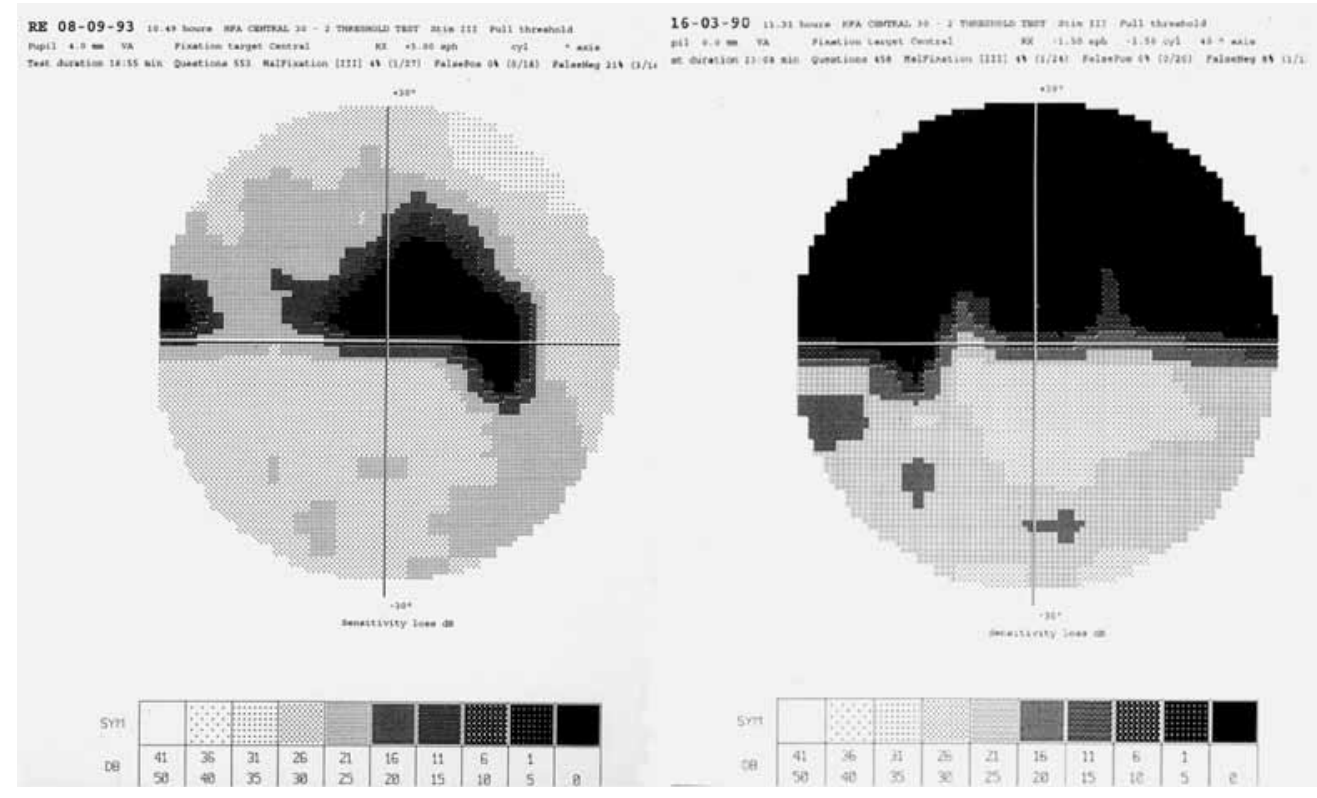

Figura 2. La campimetría a umbral de los 30 grados centrales permite identificar el escotoma asociado a la pérdida de agudeza visual. En la imagen de la izquierda se ve un escotoma centrocecal con respeto del rafe medio, en una neuritis retrobulbar del ojo derecho. A la derecha se identifica un escotoma altitudinal, en una neuropatía óptica isquémica anterior no arterítica del ojo izquierdo.

\section{DIAGNÓSTICO}

Los estudios radiológicos y de laboratorio no son necesarios si los datos clínicoexploratorios son característicos.

Los potenciales evocados visuales son útiles para la confirmación diagnóstica, especialmente en los casos atípicos. El patrón normal es una gran onda positiva a $100 \mathrm{~ms}$ (P100). En la fase aguda de la NO esta onda está disminuida en amplitud o ausente si la pérdida de agudeza visual es grave y cualquier respuesta que permanezca está retrasada. Conforme la visión 
se recupera, la amplitud crece y se obtiene una respuesta bien configurada aunque retardada. Si la remielinización es adecuada, la latencia va disminuyendo progresivamente volviendo a valores normales. Los potenciales evocados visuales pueden aportar información sobre desmielinización previa en ojos asintomáticos y así sobre el diagnóstico de EM dada la alta frecuencia en que el nervio óptico está afectado en esta enfermedad ${ }^{2}$.

Aunque no es necesaria, la confirmación diagnóstica por neuroimagen se puede obtener en la mayoría de los $\operatorname{casos}^{3}$. La tomografía computarizada es muy poco sensible para detectar la inflamación del nervio óptico. Sin embargo, la resonancia magnética (RM) del nervio óptico puede mostrar, con secuencias apropiadas, anomalías en la fase aguda en el $95 \%$ de los pacientes con NO. Es preciso incluir imágenes coronales de órbita suprimiendo la señal de la grasa orbitaria. Kupersmith y $\mathrm{col}^{4}$ han demostrado la alta sensibilidad de la RM con gadolinio (Gd) así como la correlación del realce de nervio óptico inflamado con el déficit visual; la longitud y localización de la captación no son predictivas del grado de recuperación visual. La RM cerebral tiene un papel fundamental en el diagnóstico etiológico de la NO y en el pronóstico evolutivo neurológico o conversión de síndrome desmielinizante aislado a EM.

\section{RNFL THICKNESS AVERAGE ANALYSIS}
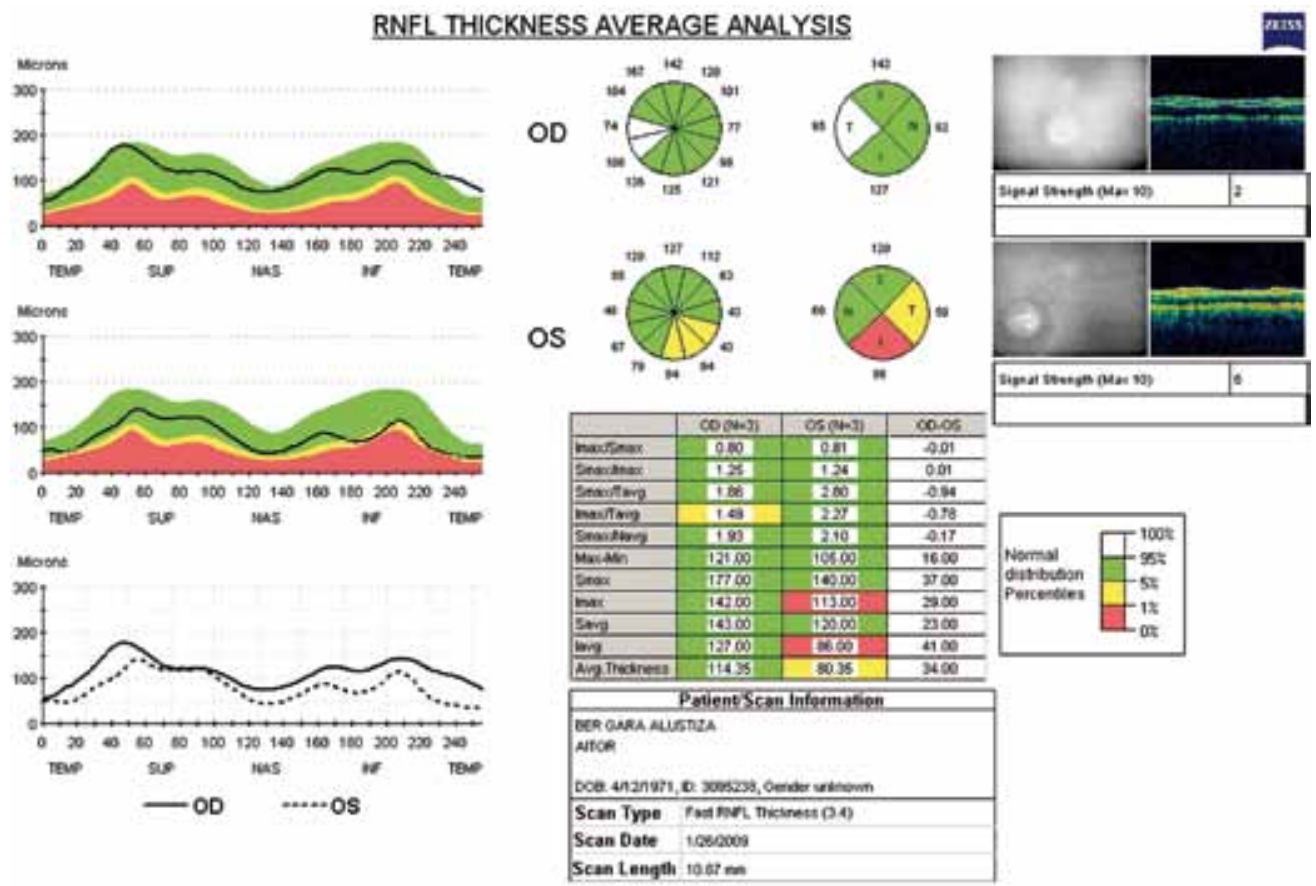

Figura 3. La tomografía óptica de coherencia de la capa de fibras peripapilares ha despertado un gran interés en el manejo de la neuritis óptica por su valor diagnóstico y pronóstico en relación con la esclerosis múltiple. Se muestra un análisis del grosor de la capa de fibras en un paciente con esclerosis múltiple remitente-recurrente que ha sufrido dos episodios de neuritis óptica en ojo izquierdo y una en ojo derecho. Se puede valorar la pérdida de grosor en la capa de fibras del haz papilomacular del ojo izquierdo. 
La tomografia de coherencia óptica (Optical coherente tomography, OCT) es una nueva técnica exploratoria diseñada para evaluar de forma cuantitativa y cualitativa el adelgazamiento de la capa de fibras nerviosas de la retina, la cabeza de nervio óptico y la mácula. Permite de forma fácil y precisa obtener imágenes del espesor de la retina y cuantificar su grosor y el de su capa más interna o capa de fibras nerviosas. Ha demostrado ser una herramienta diagnóstica útil en pacientes con NO, en la fase aguda puede ayudar a distinguir las formas que se presentan con edema de la capa de fibras nerviosas y a lo largo de la evolución a monitorizar el adelgazamiento de la misma ${ }^{5}$. La pérdida de espesor de dicha capa en la NO se debe a la destrucción de los axones del nervio óptico y se puede correlacionar con la pérdida de función visual en pruebas de sensibilidad de contraste, campimetria y de agudeza visual $^{6}$ (Fig. 3).

\section{PRONÓSTICO VISUAL}

La historia natural de la NO ha sido estudiada en múltiples trabajos en los últimos años. Uno de los ensayos más importantes es el ONTT (Optic Neuritis Treatment Trial), realizado por el Instituto Nacional de Salud Ocular de Estados Unidos que registró 389 pacientes de múltiples centros entre los años 1988 y 1991 con el objetivo inicial de valorar la eficacia del tratamiento esteroideo en la NO. El seguimiento de los pacientes durante más de 15 años ha aportado gran información sobre la evolución visual, eficacia del tratamiento y relación con $\mathrm{EM}^{7,8}$.

El pronóstico visual de la NO idiopática es excelente en el 90-95\% de los casos 3 ; el ONTT confirma que la recuperación espontánea comienza a las 3 primeras semanas después del inicio de los síntomas y continúa pasado el año ${ }^{9}$. Peores parámetros clínicos basales en cuanto a agudeza visual, sensibilidad al contraste y campos visuales son factores pronósticos negativos de evolución visual a los 6 meses, especialmente si no hay recuperación avanzada al mes ${ }^{10}$.
Se ha asociado también con mejor evolución clínica una mayor amplitud del PEV en fase aguda y lesión corta en RM de nervio óptico. El tratamiento con corticoides en fase aguda es eficaz para una recuperación más rápida de la visión pero no ha demostrado beneficio significativo a largo plazo $^{11}$.

En el seguimiento evolutivo de la NO, los pacientes pueden presentar secuelas funcionales como pérdida de visión cromática o sensibilidad al contraste e intolerancia a la luz brillante. No es inhabitual la presencia del fenómeno de Uhthoff o disminución visual transitoria tras la exposición al calor o al ejercicio. En la exploración de fondo de ojo puede aparecer atrofia óptica y en la OCT, adelgazamiento de capa de fibras nerviosas como expresión de pérdida axonal en el nervio óptico.

\section{RIESGO DE DESARROLLAR ESCLEROSIS MÚLTIPLE}

Es frecuente que la NO sea una manifestación inicial de EM y se han estudiado ampliamente los factores implicados en el desarrollo de esta enfermedad después de NO aislada. Basándose sólo en criterios clínicos, Rizzo y Lessell ${ }^{12}$ siguieron a 60 pacientes con NO típica durante 15 años encontrando que el $74 \%$ de las mujeres desarrollaron EM frente a un 34\% de varones. Después de la aparición de la RM, la presencia de anomalías de la señal de sustancia blanca cerebral en secuencias T2 sugestivas de desmielinización ha demostrado ser el mayor predictor de conversión a EM.

En el estudio Barcelona ${ }^{13}$, se incluyeron, desde 1995 a 2004, 320 pacientes con síndrome clínico aislado de los que 123 tenían neuritis óptica y fueron seguidos con RM cerebrales seriadas durante 2-3 años. El riesgo de conversión a EM definida fue del $5,9 \%$ si la RM era normal y del $55 \%$ si había lesiones.

La cohorte ONTT ha sido seguida durante 15 años $^{14}$ demostrando al cabo de este tiempo un riesgo global de EM del 
$50 \%$ con una fuerte asociación a la presencia o no de lesiones en RM cerebral inicial: la probabilidad en pacientes sin lesiones es el $25 \%$ y del $72 \%$ si hay una o más. El riesgo fue más alto en los primeros 5 años y después disminuyó aunque permanecía a lo largo de los 15 años de observación en los pacientes que tenían alteraciones en la RM basal. Entre los pacientes sin EM a los 10 años, la probabilidad de desarrollarla a los 15 fue del $32 \%$ si había lesiones iniciales y solo del $2 \%$ si la RM era normal. No hubo diferencia apreciable en el riesgo de desarrollar EM entre los 3 grupos iniciales de tratamiento a diferencia del estudio de Beck a dos años en que el grupo tratado con corticoides por vía intravenosa tenía una reducción del riesgo de un segundo episodio clínico ${ }^{15}$. Tampoco se ha encontrado relación entre el grado de discapacidad generada por la EM y el número de lesiones en la RM inicial.

Resultados similares presenta el estudio de Brex ${ }^{16}$ que recoge 71 pacientes con síndrome desmielinizante agudo donde 36 (51\%) padecían NO; durante un seguimiento de 14 años, desarrollaron EM el 19\% de los casos con RM normal al incluirse en el ensayo y el $88 \%$ de los que presentaban al menos una lesión. A diferencia de los resultados del ONTT, el grado de discapacidad causada por la EM estaba relacionado con el volumen lesional en la RM de los primeros años.

Además de las lesiones de RM se han implicado otros parámetros en la progresión a EM. En un estudio realizado en Suecia se registraron 147 pacientes con NO entre los años 1990 y 1995, se analizó la presencia de múltiples variables clínicas y ambientales además de la RM y test de laboratorio. Resultaron fuertes predictores de desarrollo de EM, además de 3 lesiones o más en RM, la presencia de bandas oligoclonales en LCR ${ }^{17}$. Kheradvar y col $^{18}$ estudiaron en población iraní la influencia genética evaluando antígenos HLA en 55 pacientes con NO y siguieron su evolución durante 4 años. Estos auto- res encontraron discrepancia en el número de alelos HLA de clase II entre los que desarrollan EM y los que no. El alelo DR2 parece ser un factor de susceptibilidad con un $12 \%$ mayor de riesgo en el grupo que lo presenta. También los alelos HLA de clase I A23 y B1 se asocian con mayor evolución a EM. Los potenciales evocados motores y somatosensoriales anormales pueden ser también factores predictivos aunque su sensibilidad es muy inferior a la RM; se ha postulado que pueden jugar un papel para definir pacientes con peor pronóstico y más rápida conversión a $\mathrm{EM}^{19}$.

\section{DIAGNÓSTICO DIFERENCIAL}

Hay otras patologías de nervio óptico que pueden simular NO inflamatoria y que precisan estudios de laboratorio o radiológicos para su diagnóstico (Tabla $1)^{20}$. La neuropatía óptica anterior isquémica cursa también con pérdida de visión monocular, pero la presentación es más brusca y es originada por infarto del disco óptico secundario a insuficiencia vascular en el territorio de las arterias ciliares posteriores. Difiere de la NO en la edad de presentación ya que esta entidad aparece sobre todo en sujetos mayores de 50 años y además presentan en la exploración fundoscópica edema y hemorragias de disco óptico (Fig. 4). Por otra parte, raramente cursa con dolor ocular y la recuperación a largo plazo es mínima. Según sea el mecanismo patogénico de la isquemia se clasifica en arterítica y no arterítica ${ }^{3}$.

Las neuropatías tóxicas ocurren como efecto secundario de radiación, tóxicos como el metanol, y múltiples fármacos que por diferentes mecanismos pueden ocasionar neuropatía óptica: etambutol, amiodarona, ciclosporina, vitamina A o isoniacida. También déficits nutricionales, especialmente por carencia de una o más vitaminas del grupo B y ácido fólico pueden causar daño del nervio óptico. 
Tabla 1. Diagnóstico diferencial de neuritis óptica.

\begin{tabular}{|c|c|}
\hline Etiología & Enfermedad \\
\hline \multirow{2}{*}{ Isquémica } & NOAI* $^{*}$ arterítica \\
\hline & NOAI no arterítica \\
\hline \multirow{3}{*}{ Tóxico/metabólica } & Radiación \\
\hline & Fármacos y tóxicos \\
\hline & Carencial \\
\hline \multirow{2}{*}{ Infecciosa } & Sífilis, tuberculosis, Lyme \\
\hline & $\mathrm{CMV}^{* *}$, hongos, sinusitis paranasales \\
\hline \multirow{3}{*}{ Parainfecciosa/Autoinmune } & Postinfecciosa \\
\hline & Postvacunal \\
\hline & Paraneoplásica \\
\hline \multirow{5}{*}{ Neoplásica } & Tumores de nervio óptico \\
\hline & Masa intracraneales \\
\hline & Metástasis \\
\hline & Abscesos \\
\hline & Aneurismas \\
\hline Hereditaria & Neuropatía óptica de Leber \\
\hline \multirow{5}{*}{ Inflamatoria/vasculítica } & Sarcoidosis \\
\hline & Granulomatosis de Wegener \\
\hline & Lupus \\
\hline & Sjögren \\
\hline & Behçet \\
\hline \multirow{3}{*}{ Desmielinizante } & Idiopática \\
\hline & Esclerosis múltiple \\
\hline & Neuromielitis óptica \\
\hline
\end{tabular}

${ }^{*}$ NOAI: Neuritis óptica anterior isquémica ** CMV: Citomegalovirus
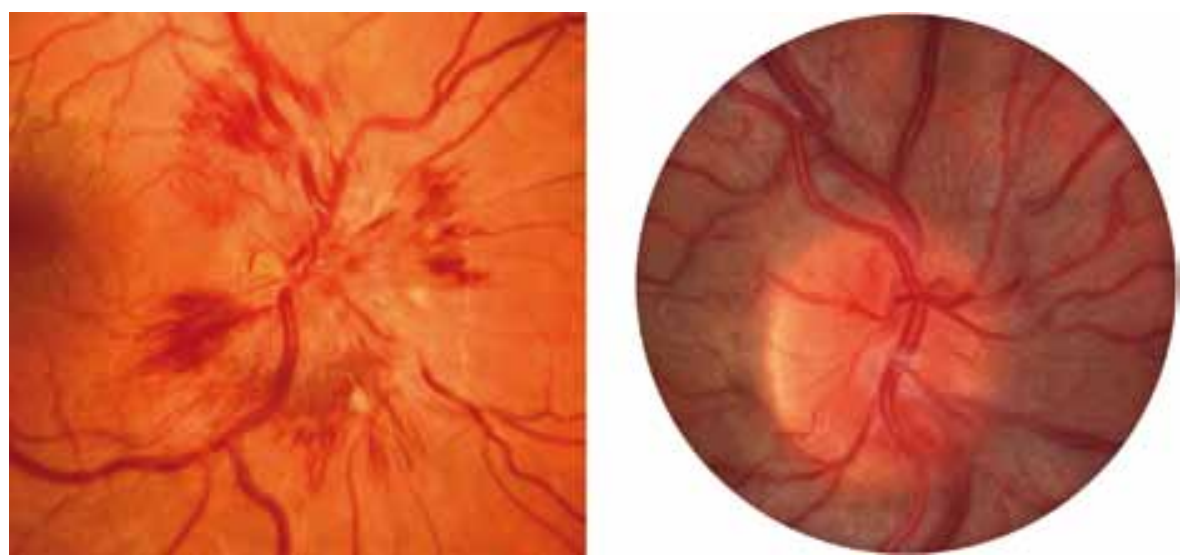

Figura 4. La exploración del nervio óptico servirá para plantear un diagnóstico diferencial. Aunque en la neuritis retrobulbar la exploración del nervio óptico será normal, otras neuropatías mostrarán alteraciones características. En la imagen de la izquierda, se aprecia un nervio óptico congestivo con múltiples hemorragias en llama, pérdida de excavación y bordes y exudados algodonosos compatibles con una neuropatía óptica isquémica anterior. A la derecha, la papila aparece congestiva con pérdida de la excavación y bordes nasales e inferiores borrosos sugerentes de una papilitis. 
En la neuropatía óptica infecciosa, la inflamación del nervio óptico es el resultado de infección directa por virus o bacterias. Según se trate de personas inmunocompetentes o inmunocomprometidos se debe pensar en diferentes agentes causales: sífilis, enfermedad de Lyme, enfermedad por arañazo de gato (Bartonella henselae) y virus Herpes Simple aparecen en inmucompetentes; tuberculosis, toxoplasmosis y citomegalovirus son más prevalentes en inmunodeficientes. En ocasiones es difícil distinguirla de la neuritis óptica parainfecciosa en la que el daño del nervio óptico es secundario a la respuesta inmune del paciente por infección sistémica o del propio sistema nervioso central. Aparece de una a tres semanas después de la infección y es más frecuente en niños. Generalmente ocurre en el contexto de encefalomielitis aguda diseminada.

Infecciones de la proximidad como sinusitis paranasales o mucocele pueden ocasionar neuropatía óptica de causa inflamatoria o compresiva ${ }^{21}$. Neoplasias primarias del nervio óptico o meningiomas de base craneal, diseminación de tumores sistémicos, adenoma pituitario, procesos óseos o aneurismas originan también neuropatía óptica por compresión o infiltración (Fig. 5). Entre las causas hereditarias la neuropatía óptica de Leber es la más común; a menudo es bilateral y cursa con pérdida visual más severa.

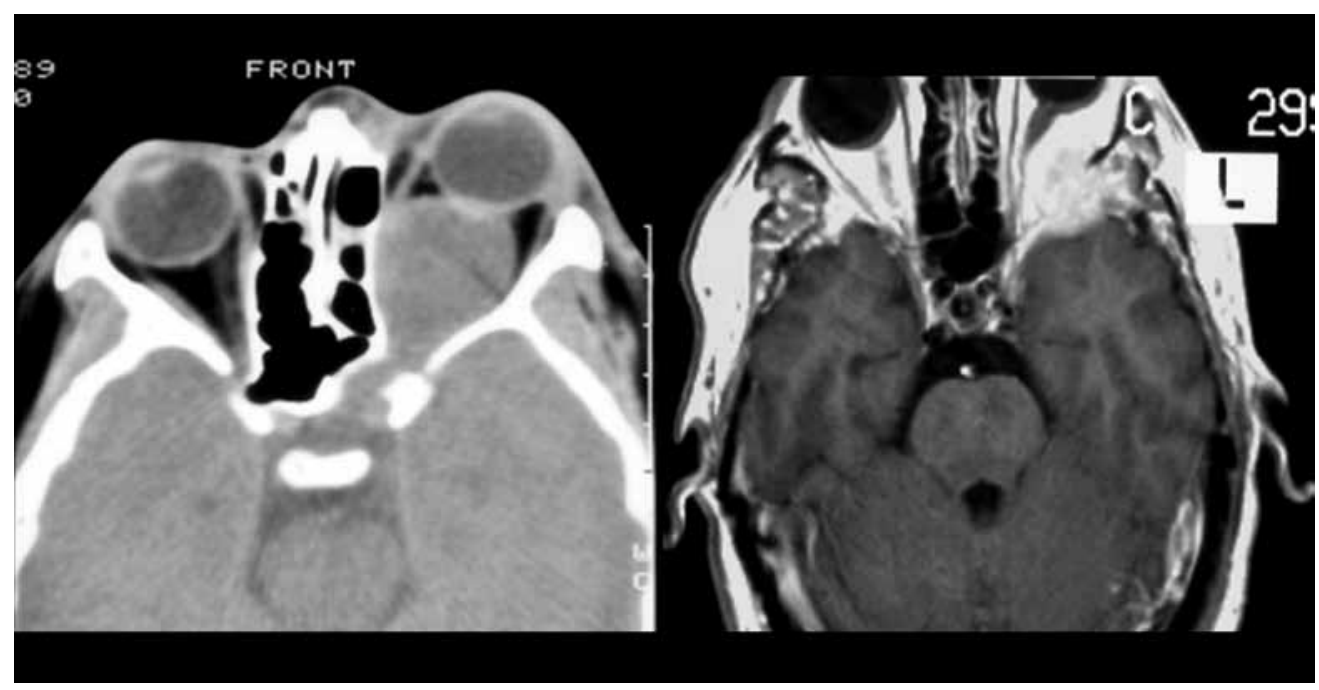

Figura 5. Las técnicas de imagen CT y RM a nivel orbitario con cortes finos de 3 mm permiten identificar el nervio óptico, ayudando a diagnóstico diferencial de la neuritis óptica. En el CT se observa un engrosamiento marcado del nervio óptico, con ocupación de la cavidad orbitaria y exoftalmos secundaria a glioma del nervio óptico. En la RM se identifica una masa a nivel del ala mayor del esfenoides que produce una neuropatía óptica compresiva por un mieloma múltiple.

Es bien conocido que enfermedades del tejido conectivo pueden caracterizarse por una amplia afectación del sistema nervioso central; además, síndromes neurológicos aislados pueden ser también la primera manifestación de lupus eritematoso sistémico, síndrome antifosfolípido, Sjögren,
Behçet o de enfermedades inflamatorias sistémicas como sarcoidosis o Wegener.

La neuropatía óptica autoinmune en el lupus eritematoso sistémico es una manifestación infrecuente aunque bien conocida, suele ser unilateral y aparece de forma aislada o asociada a otras manifestaciones neu- 
rológicas. Los mecanismos implicados en la patogénesis de la afectación neurológica son: interacción directa de autoanticuerpos con autoantígenos en membranas celulares neuronales, isquemia mediada por anticuerpos antifosfolípidos, microtrombosis y vasculopatía no inflamatoria y producción local de citoquinas inductoras de citotoxicidad neuronal ${ }^{22}$. Para diferenciar la neuropatía óptica secundaria a lupus eritematoso sistémico de la asociada a EM son útiles la RM craneal, estudio del LCR y detección de autoanticuerpos en suero y LCR.

En el síndrome de Sjögren hay participación neurológica en el 25-30\% de los casos manifestada como desmielinización, disfunción autonómica, ictus, mielopatía o neuropatía óptica autoinmune. En ocasiones, sobre todo si la clínica neurológica precede al resto de anomalías inmunológicas, puede ser similar a la EM. La determinación de los anticuerpos característicos Ro (SS-A) y La (SS-B) puede ayudar al diagnóstico.

\section{Sarcoidosis}

Es una enfermedad granulomatosa multisistémica de etiología desconocida aunque se relaciona con factores genéticos y ambientales. Las manifestaciones clínicas más frecuentes son linfadenopatías mediastínicas a nivel pulmonar y en piel; hay afectación oftalmológica en el $25 \%$ de los casos, aunque predomina en el segmento anterior. La neurosarcoidosis se limita al $5 \%$ de los casos y cursa con gran heterogeneidad clínica. Lo más habitual es la participación de leptomeninges y pares craneales, especialmente nervio facial y óptico, también puede ocurrir a nivel cerebral, medular o sistema nervioso periférico. La neuropatía óptica sarcoidea ha sido la manifestación más común en algunas series de neurosarcoidosis ${ }^{23}$. Los mecanismos responsables de la disfunción del nervio óptico son variables, puede haber papiledema originado por hipertensión intracraneal secundaria a hidrocefalia $o$ lesiones granulomatosas en parénquima, compresión o infiltración granulomatosa de nervio óptico; la neuritis retrobulbar puede ser similar a la hallada en EM. En la exploración se pueden encontrar signos de uveítis anterior, papilitis, papiledema, atrofia óptica o granulomas (Fig. 6).

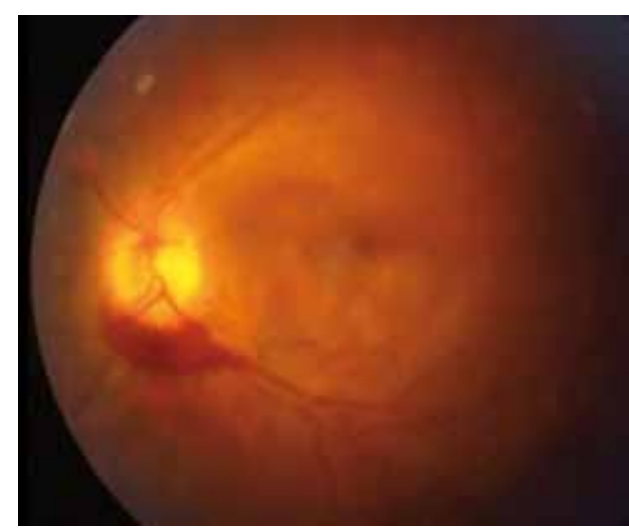

Figura 6. Se observa una borrosidad del vítreo por aumento de la celularidad de éste, vitritis; un discreto envainamiento vascular, lo que sugiere una vasculitis asociada, y una hemorragia peripapilar inferior. La papila pese a la dificultad para apreciar detalles tiene una apariencia normal. El cuadro corresponde a una sarcoidosis ocular con neuritis, vitritis y vasculitis.

El diagnóstico definitivo se basa en la demostración histológica del tejido granulomatoso por lo que puede ser especialmente difícil si la enfermedad debuta con clinica neurológica. La realización de gammagrafia con galio, RM o tomografía de emisión de positrones (PET) pueden ser útiles para la búsqueda de sarcoidosis sistémica y estudio de otros órganos afectados.

\section{NEURITIS ÓPTICA BILATERAL}

Es relativamente frecuente que la neuritis óptica afecte de forma secuencial a ambos ojos tanto en la EM como en otras enfermedades inflamatorias y desmielinizantes. Más inusual es la neuritis óptica bilateral simultánea aunque ocurre con frecuencia en algunas patologías como encefalomielitis aguda diseminada (EAD), neuropatía óptica de Leber, neuropatía óptica inflamatoria recurrente crónica y neuromielitis óptica (NMO). 


\section{Encefalomielitis aguda diseminada}

La EAD es una enfermedad inflamatoria y desmielinizante de sistema nervioso central, tradicionalmente monofásica, que cursa casi siempre con encefalopatía a la que se asocian síndromes focales o multifocales incluyendo neuritis óptica y mielitis. La neuroimagen es importante para el diagnóstico. En la RM se identifican en secuencias T2 y FLAIR áreas de hiperseñal grandes, múltiples y asimétricas en sustancia blanca central y subcortical, unión cortico-subcortical y con frecuencia en sustancia gris de tálamo y ganglios basales. En ocasiones puede cursar de forma recurrente cuando un nuevo episodio desmielinizante acontece meses después del evento inicial o multifásica cuando hay recaídas que afectan a nuevas áreas de $\mathrm{SNC}^{24}$. El LCR revela pleocitosis e hiperproteinorraquia; no es frecuente la presencia de bandas oligloconales a diferencia de la EM. Ocurre más en niños que en adultos y en algunas series se ha descrito predominancia masculina.

Dentro de las manifestaciones neurológicas la neuritis óptica es muy habitual, en muchos casos bilateral. La EAD se ha asociado con múltiples infecciones previas, especialmente víricas, u otros desencadenantes inmunológicos como vacunaciones aunque en ocasiones no hay antecedentes de infección y no es un criterio necesario para su diagnóstico. Se acepta que es un proceso inmune mediado de SNC independiente del agente desencadenante.

Aún no se han definido criterios diagnósticos fiables ni hay biomarcadores específicos de EAD, por tanto, ante un episodio monofásico es difícil establecer un diagnóstico de certeza y diferenciar esta entidad de otras enfermedades desmielinizantes de sistema nervioso central ${ }^{25}$.

\section{Neuropatía óptica de Leber}

Es una neuropatía óptica hereditaria producida por mutaciones puntuales en el ADN mitocondrial que causa pérdida bilateral aguda o subaguda de visión cen- tral; suele ser de comienzo asimétrico con breve intervalo entre ambos ojos. La afectación monocular es excepcional. Es más frecuente y el inicio es más precoz en varones; la edad de comienzo es de 15 a 35 años. El deterioro visual es rápido al inicio, aunque puede ocurrir progresivamente en un mes y el deterioro es permanente, rara vez hay mejoría o recuperación. Puede cursar con dolor con los movimientos oculares y fenómeno de Uhthoff aunque con menor frecuencia que la neuritis óptica desmielinizante. En el fondo de ojo se observa una microangiopatía telangiectásica peripapilar e inflamación del disco óptico que puede verse ya en estadio presintomático o en familiares asintomáticos, después de la pérdida visual la retina aparece pobre en capilares con arteriolas atenuadas y atrofia de nervio óptico. El déficit campimétrico es central, centrocecal o bitemporal. La visión cromática está muy afectada. La NO puede ocurrir de forma aislada o asociarse a otros síntomas de disfunción neurológica e incluso se ha descrito un trastorno similar a la EM con lesiones desmielinizantes en sustancia blanca y bandas oligoclonales en $\mathrm{LCR}^{26}$, esta forma ocurre con más frecuencia en mujeres aunque también hay casos publicados en varones ${ }^{27}$.

\section{Neuropatía óptica inflamatoria recurrente crónica}

CRION, acrónimo de chronic relapsing inflammatory optic neuropathy es una neuropatía inflamatoria desmielinizante caracterizada por la afectación bilateral simultánea o secuencial de instauración subaguda, con dolor intenso y persistente y rápida respuesta al tratamiento esteroideo pero con recaídas al suprimirlo ${ }^{28}$. Puede presentarse después de una infección vírica o vacunación, especialmente en niños. La RM muestra inflamación del nervio óptico que se realza con contraste siendo el cerebro normal. El déficit visual es mayor que en otros casos de NO desmielinizante y en la campimetría puede haber defectos altitudinales o escotoma central. Lo que diferencia esta neuritis de la asociada a 
EM es la afectación bilateral, la mayor intensidad del dolor y de la pérdida visual, la ausencia de lesiones en sustancia blanca en RM craneal, la menor frecuencia de bandas oligoclonales en LCR y su corticodependencia. A menudo se precisa tratamiento inmunosupresor prolongado para prevenir recaídas y modificar el curso de la enfermedad. Histológicamente se trata de una enfermedad granulomatosa similar a la neurosarcoidosis aunque focal, sin evidencia de diseminación de lesiones después de un largo seguimiento clínico.

Otra entidad que cursa con neuritis óptica bilateral y recurrente es la NMO que difiere de la CRION por la presencia de anticuerpos antiaquaporina y extensión de la enfermedad a nivel de SNC especialmente en forma de mielitis ${ }^{29}$.

\section{Neuromielitis óptica}

La NMO es una enfermedad desmielinizante e idiopática de SNC descrita por Devic en el siglo XIX. Inicialmente se describió como un síndrome monofásico, variante de la EM, caracterizado por neuritis óptica bilateral y mielitis transversa que ocurrían de forma simultánea o en rápida sucesión con ausencia de afectación cerebral. En la actualidad, se considera una enfermedad desmielinizante recurrente con características clínicas, radiológicas, histopatológicas e inmunológicas bien definidas que permiten diferenciarla de la EM. Clínicamente es una enfermedad que cursa con ataques recurrentes, con más frecuencia que simultáneos, de neuritis óptica y mielitis transversa extensa longitudinalmente. El curso de la enfermedad es monofásico en el $20 \%$ y recurrente en el $80 \%$ de los casos. La evolución de la NMO no tratada es peor que la de la EM con déficit visual permanente y dificultad para la deambulación a los 5 años del inicio clínico en el $50 \%$ de los casos. El LCR de la NMO muestra pleocitosis de predominio polimorfonuclear durante recaídas en el $25 \%$ de los casos y baja frecuencia de bandas oligoclonales a diferencia de la EM donde la celularidad es escasa (inferior a 50 células) y hay bandas oligoclonales en el 85-90\% de los pacientes. La RM medular y cerebral son de gran valor en el diagnóstico de la NMO; en la fase aguda de mielitis transversa longitudinal hay alteración de la señal en T2 que afecta a la médula a lo largo de 3 segmentos vertebrales o más, en la EM los segmentos lesionados son cortos. Además, la RM craneal puede ser normal o inespecífica al comienzo de la NMO pero en fases avanzadas presenta lesiones que típicamente se localizan en diencéfalo (tálamo e hipotálamo) y regiones adyacentes al tercero y cuarto ventrículos; solo en $10 \%$ de los casos la RM cumplirá criterios de EM.

Los estudios patológicos de la NMO muestran lesiones con infiltrados inflamatorios densos predominando los eosinófilos y granulocitos, desmielinización y necrosis en la sustancia blanca y gris. Los vasos intralesionales están engrosados y hialinizados. Es característico un patrón vasculocéntrico con depósitos de inmunoglobulinas y activación de complemento que difiere de los patrones descritos en la EM clásica y sugiere un papel patogénico de la inmunidad humoral con el antígeno diana en el área perivascular ${ }^{30}$. En 2004 Lenon y col${ }^{31}$ identificaron un autoanticuerpo sérico específico denominado IgG-NMO cuya diana es la aquaporina 4 (AQP4), proteína de canal de agua más abundante del SNC. La distribución de AQP4 en el cerebro normal, nervio óptico y médula espinal semeja el patrón vasculocéntrico del depósito de inmunocomplejos observado en las lesiones de NMO; la inmunotinción de IgG-NMO a este nivel y la pérdida de AQP4 en sitios de intensos depósitos de inmunocomplejos apoya el papel patogénico de este anticuerpo ${ }^{32}$. Además, la mejoría de las recaídas de NMO con plasmaféresis y la ausencia de bandas oligoclonales en LCR sugieren también que existe un anticuerpo sérico circulante patogénico en oposición a un anticuerpo patogénico generado dentro de SNC.

La detección de este anticuerpo, con una sensibilidad del 73\% y especificidad del $91 \%$, ha llevado a revisar los criterios diagnósticos de NMO que ayudan a distinguir esta enfermedad de formas de presen- 
tación óptico-espinales de EM (Tabla 2). Este biomarcador ha sido también identificado en otras enfermedades relacionadas y contribuye a definir entidades del espectro $\mathrm{NMO}^{33}$. Se han detectado IgG-NMO en el $50 \%$ de pacientes con mielitis transversa longitudinal y en $25 \%$ de pacientes con neuritis óptica recurrente o simultánea; su presencia se asocia a un alto riesgo de recurrencia o conversión a NMO.

Tabla 2. Criterios diagnósticos de neuromielitis óptica (2006).

\begin{tabular}{l}
\hline Criterios absolutos \\
\hline - Neuritis óptica \\
- Mielitis aguda \\
\hline Criterios de apoyo \\
- RM cerebral negativa al inicio \\
- RM de médula espinal con alteración de la señal en T2 en 3 ó más segmentos vertebrales \\
- Presencia de anticuerpos IgG NMO
\end{tabular}

Criterios requeridos: todos los absolutos y al menos 2 de los criterios de apoyo.

\section{MANEJO CLÍNICO Y TERAPÉUTICO DE LA NEURITIS ÓPTICA INFLAMATORIA}

El diagnóstico de la NO inflamatoria es fundamentalmente clínico y los tests de laboratorio van dirigidos a aquellos casos atípicos en los que se deba buscar una causa alternativa. Se considera NO atípica (Tabla 3$)^{20}$, si la edad de presentación es inferior a 16 años y en mayores de 45, inicio simultáneo bilateral, ausencia de dolor ocular, falta de mejoría después de
4-6 semanas del comienzo clínico, pérdida progresiva visual más allá de 2 semanas después del comienzo de los síntomas o presencia en fundoscopia de hemorragias retinianas o exudados maculares (estrella macular). En estos pacientes, debe hacerse una valoración más exhaustiva; la analítica de sangre debería incluir, junto a las determinaciones rutinarias, anticuerpos antinucleares, enzima angiotensina convertasa, anticuerpos anticitoplasma de neutrófilos, vitamina B12, ácido fólico y tiamina, serología de sífilis y radiografía de tórax.

Tabla 3. Datos de neuritis óptica atípica.
1. Edad de presentación $<16$ años ó $>45$ años
2. Inicio simultáneo bilateral
3. Ausencia de dolor ocular
4. Falta de mejoría después de 4-6 semanas del comienzo
5. Pérdida progresiva visual más allá de 2 semanas
6. Hemorragias retinianas o exudados maculares en fondo de ojo

La RM craneal es indispensable en todos los casos, en los atípicos buscando otra causa de neuropatía óptica y en los típicos para valorar el riesgo de progresión a EM; la RM o la tomografía computarizada orbitaria sólo son precisas en la NO atípica.
La realización de punción lumbar no es necesaria en pacientes con forma típica aunque como ya se ha comentado la presencia en LCR de bandas oligoclonales y síntesis intratecal de IgG son también predictores de riesgo de conversión a EM. Debería es- 
tudiarse el LCR en casos seleccionados de NO atípica, especialmente en NO bilateral o cuando haya sospecha de etiología infecciosa o enfermedad inflamatoria sistémi$\mathrm{ca}^{9}$.

El tratamiento agudo tradicional de la NO son los corticoides; determinar si esteroides intravenosos o por vía oral modifican la evolución visual de pacientes con NO inflamatoria fue el objetivo primario del ONTT cuyos resultados definitivos han sido publicados después de 20 años de seguimiento ${ }^{34}$. No debe indicarse prednisona vía oral puesto que no es eficaz en la rapidez de la recuperación ni en la evolución a largo plazo; en los resultados preliminares del ONTT se observó una mayor tasa de recurrencia que no se confirmó en estudios posteriores. Aunque su uso no está justificado y ha declinado en los últimos años, todavía es frecuente su utilización por algunos oftalmólogos y neurólogos pensando que ayudan a la recuperación visual ${ }^{35}$. Los esteroides por vía intravenosa aceleran la recuperación de la visión pero no hay diferencias significativas finales en agudeza visual, campimetría, visión cromática ni sensibilidad al contraste. Por tanto, la decisión del tratamiento agudo debe individualizarse en base al riego/beneficio si bien en general son bien tolerados. La pauta habitual es de 1 gramo de metilprednisolona intravenoso al día durante 3 días.

Un ensayo randomizado sobre la eficacia de inmunoglobulinas intravenosas en NO no demostró tampoco mejoría a largo plazo en los déficits visuales.

Aunque en casos aislados de NO resistentes a esteroides se ha apreciado mejoría funcional tras plasmaféresis, se precisan ensayos randomizados para valorar el papel terapéutico de esta técnica.

El tratamiento crónico de NO debe ir dirigido a aquellos pacientes con alto riesgo de progresión a EM; las anomalías en la RM son el factor predictor más útil en el reconocimiento de estos casos. El seguimiento clínico y radiológico es esencial para probar la diseminación en tiempo y espacio, criterios requeridos para el diagnóstico de EM.
Las diferentes terapias modificadoras de la enfermedad ahora disponibles han demostrado eficacia clínica para reducir los brotes, minimizar las lesiones de RM y mejorar la calidad de vida ${ }^{36}$. Varios ensayos clínicos recientes han evaluado su utilidad en el síndrome clínico aislado como la NO para retrasar la conversión a EM. CHAMPS ${ }^{37}$ fue un ensayo controlado y randomizado de interferon beta-1a (Avonex) que incluyó 383 pacientes con dicho síndrome, de los cuales 192 tenían neuritis óptica aislada. Se incluyeron sólo aquellos que tenían al menos 2 anomalías de la señal de RM cerebral de 3 ó más mm de diámetro. El principal resultado de dicho estudio fue que el fármaco podía disminuir la conversión a EM en torno al 50\% en estos pacientes de alto riesgo; el estudio de seguimiento CHAMPIONS ${ }^{38}$ mostró que estos resultados se mantuvieron a los 5 años. En el estudio ETOMS ${ }^{39}$ con 308 pacientes que presentaron síndrome clínico aislado monofocal o multifocal, 98 pacientes tenían neuritis óptica; fueron criterios de inclusión de RM la presencia de 4 ó más lesiones y se trataron con interferón beta-1a subcutáneo (Rebif) o placebo. A los 2 años el grupo tratado con interferón tenía también una menor probabilidad de desarrollar EM comparado con el placebo (34\% versus $45 \%$ ). En el ensayo de Interferón beta 1b (BENEFIT) ${ }^{40}$ participaron 487 pacientes con síndrome clínico aislado de los que 80 tenían neuritis óptica y al menos 2 lesiones en RM craneal. Después de 2 años, $28 \%$ de pacientes del grupo tratado con interferón beta-1 b tenían criterios de EM clínicamente definida y $45 \%$ de los incluidos en el grupo placebo. El ensayo PRECISE randomizó pacientes con síndrome clínico aislado y RM anormal a acetato de glatiramer (Copaxone) o placebo. Los que recibieron el fármaco desarrollaron EM en el $25 \%$ de los casos frente al $43 \%$ de los del grupo placebo; además se apreció un descenso del $61 \%$ en la aparición de nuevas lesiones en RM.

Estos estudios demuestran los beneficios del uso precoz de tratamientos inmunomoduladores para disminuir la actividad clínica y radiológica de la patología desmielinizante. 


\section{CONSIDERACIONES FINALES}

La neuritis óptica inflamatoria es la causa más frecuente de pérdida visual aguda en adultos jóvenes. Es un proceso inflamatorio y desmielinizante de nervio óptico con buen pronóstico visual.

Es necesario conocer las características clínicas y exploratorias habituales realizando un estudio etiológico más exhaustivo en las formas atípica y NO bilaterales.

El tratamiento de la NO inflamatoria debe realizarse por vía intravenosa y debe ser individualizado teniendo en cuenta que no modifica a largo plazo el pronóstico visual funcional.

Un alto porcentaje de pacientes con NO desarrollarán Esclerosis Múltiple en años posteriores. Es preciso identificar los casos con alto riesgo de conversión para indicar tratamiento inmunomodulador y así mejorar la historia natural de la NO.

$\begin{array}{ll}\text { Anexo: } & \text { Abreviaturas utilizadas } \\ \text { NO: } & \text { Neuritis óptica } \\ \text { EM: } & \text { Esclerosis Múltiple } \\ \text { ONTT: } & \text { Optic Neuritis Treatment Trial } \\ \text { OCT: } & \text { (Optical coherente tomography) Tomo- } \\ & \text { grafía de coherencia óptica } \\ \text { SCA: } & \text { Síndrome clínico aislado } \\ \text { LCR: } & \text { Líquido cefalorraquídeo } \\ \text { AV: } & \text { Agudeza visual } \\ \text { PEV: } & \text { Potenciales evocados visuales } \\ \text { TC: } & \text { Tomografía computarizada } \\ \text { RM: } & \text { Resonancia Magnética } \\ \text { Gd: } & \text { Gadolinio } \\ \text { CFN: } & \text { capa de fibras nerviosas } \\ \text { BOC: } & \text { bandas oligoclonales } \\ \text { HLA: } & \text { Antígeno leucocitario humano } \\ \text { NOAI: } & \text { Neuropatía Óptica Anterior Isquémica } \\ \text { VHS: } & \text { Virus Herpes Simple } \\ \text { EAD: } & \text { Encefalomielitis aguda diseminada } \\ \text { LES: } & \text { Lupus eritematoso sistémico } \\ \text { SS: } & \text { Síndrome de Sjögren } \\ \text { CRION: } & \text { Neuropatía óptica inflamatoria recurren- } \\ & \text { te crónica } \\ \text { NMO: } & \text { Neuromielitis óptica } \\ \text { SNC: } & \text { Sistema nervioso central } \\ \text { AQP4: } & \text { Aquaporina } 4\end{array}$

\section{BIBLIOGRAFÍA}

1. Miller NR, Newman NJ. Optic Neuritis. En: Wals and Hoyt's-Clinical Neuro-Opthalmology 1999, 5th Edition.
2. Miller D, McDonald I, Smith K. The diagnosis of multiple sclerosis. En: Compston A, Confavreaux C, Lassmann $\mathrm{H}$ et al. McAlpine's Multiple Sclerosis 2006, 4th edition.

3. Purvin V, KaWASAKI A. Neuro-ophthalmic emergencias for the neurologist. Neurologist 2005; 11: 195-233.

4. Kupersmith MJ, Alban T, Zeiffer B, Lefton D. Contrast-enhanced MRI in acute optic neuritis: relationship to visual performance. Brain 2002; 125: 812-822.

5. Oreja Guevara C, Noval S. La tomografía de coherencia óptica en la neuritis óptica y esclerosis múltiple. Rev Esp Esclerosis múltiple 2008, Vol I (6): 22-26.

6. Costello F, Hodge W, Pan YI. Eggenberger E, Coupland S. KARDON RH. Tracking retinal nerve fiber layer loss after optic neuritis: a prospective study using optical coherente tomography. Multiple Esclerosis 2008; 14: 893-905.

7. Beck RW, Cleary PA, Anderson MM JR, Keltner JL; Shults WT, Kaufman DI et al. A randomized, controlled trial of corticosteroids in the treatment of acute optic neuritis. N Engl J Med 1992; 326: 581-588.

8. Optic Neuritis Study Group. Neurologic impairment 10 years alter optic neuritis. Arch Neurol 2004; 61: 1386-1389.

9. Atkins E, Biousse V, Newman NJ. Optic neuritis. Semin Neurol 2007, 27: 211-220.

10. Kupersmith MJ, Gal RL, Beck RW, Xing D, Miller N. Visual function at baseline and 1 month in acute optic neuritis: predictors of visual outcome. Neurology 2007; 69: 508-514.

11. Brusaferri F, CANDElise F. Steroids for multiple sclerosis and optic neuritis: a meta-analysis of randomized controlled clinical trials. J Neurol 2000; 247: 435-442.

12. Rizzo JF, LESSELL S. Risk of developing multiple sclerosis after uncomplicated optic neuritis: a long-term prospective study. Neurology 1988; 38: 185-714.

13. Tintoré M, Rovira A, Rio J, Nos C, Grivé E, Téllez $\mathrm{N}$ et al. Is optic neuritis more benign than other first attacks in multiple sclerosis? Ann Neurol 2005; 57: 210-215.

14. The Optic Neuritis Study Trial. Multiple sclerosis risk alter optic neuritis. Final optic neuritis treatment trial follow-up. Arch Neurol 2008; 65 : 727-732.

15. Beck RW, Cleary PA, Trobe JD, Kaufman DI, KuPERSMITH MJ, PATY DW et al. The effect of corticosteroids for acute optic neuritis on the subsequent development of multiple sclerosis. N Eng J Med 1993; 329: 1764-1769. 
16. Brex PA, Ciccarelli O, O'Riordan JL, Sailer M, Thompson AJ, Miller DH. A longitudinal study of abnormalities on MRI and disability from multiple sclerosis. N Engl J Med 2002; 346: 158-164.

17. Jin YP, De Pedro-Cuesta J, Huang YH, Söderström, M. Predicting multiple sclerosis at optic neuritis onset. Multiple Sclerosis 2003; 9: 135-141.

18. Kheradvar A, Tabassi AR, Nikbin B, Khosravi F, NARoueynejad M, Moradi B et al. Influence of HLA on progresión of optic neuritis to MS: results of a tour-year follow-up study. Multiple Sclerosis 2004; 10: 526-531.

19. Simó M, BarSi P ANd ArÁnYI Z. Predictive role of evoked potencial examinations in patients with clinically isolated optic neuritis in Light of the revised McDonald criteria. Multiple Sclerosis 2008; 14: 472-478.

20. Abou ZeId N and Bhatti MT. Acute inflammatory demyelinating optic neuritis. Neurologist 2008;14: 207-223

21. Cestari DM, Metson RB, Cunnane ME, Faquin WC. Case records of the Massachusetts General Hospital. Case 40-2008. A 26-year-old man with blurred vision. N Engl J Med 2008; 359: 2825-2833.

22. Cikes N, Bosnic D, Sentic M. Non-MS autoinmune demyelination. Clin Neurol Neurosur 2008; 110: 905-912.

23. ZAJICEK JP. Neurosarcoidosis. Curr Opin Neurol 2000; 13: 323-325.

24. Tenembaum S, Chitnis T, Ness J, Hahn JS for the Internacional Pediatric MS Study Group) Acute disseminated encephalomyelitis. Neurology 2007; 68 (Suppl2): S23-S36.

25. Young NP, Weinshenker BG, Lucchinetti CF. Acute disseminated encephalomyelitis: current understanding and controversias. Semin Neurol 2008; 288: 84-94.

26. Harding AE, Sweeney MG, Miller DH, Mumford CJ, Kellar-Wood H, Menard D et al. Occurrence of multiple sclerosis-like illness in women who have a Leber's hereditary optic neuropathy mitochondrial DNA mutation. Brain 1992; 115: 978-989.

27. Pato-Pato A, Cimas-Hernando I, Lorenzo-GonzÁLEZ JR. Neuropatía óptica de Leber: caso clínico. Rev Neurol 2006; 42: 22-24.

28. Kidd D, Burton B, Plant GT, Gram. EM. Chronic relapsing inflammatory optic neuropathy (CRION). Brain 2003; 126: 276-284.

29. Pérez-Díaz H, CaSAdo-Chocan JL, Ucles-SÁnchez A, SÁız A. Neuropatía óptica inflamatoria re- currente crónica (CRION) sin detección de Acs IgG-NMO. Neurología 2007; 22: 553-555.

30. Piтtock SJ. Neuromyelitis optica: a new perspectiva. Sem Neurol 2008; 28 (1): 95-104.

31. Lennon VA, Wingerchuck DM, Kryzer TJ, Pittock SJ, LuCChinetTi CF, FuJihara K et al. A serum autoantibody marker of neuromyelitis optica: distinction from multiple sclerosis. Lancet 2004; 364: 2106-2112.

32. Roemer SF, Parisi JE, Lennon VA, Benarroch EE, Lassmann H, Bruck W et al. Pattern-specific los of aquaporin-4 immunoreactivity distinguishes neuromyelitis optica from multiple sclerosis. Brain 2007; 130: 1194-1205.

33. Wingerchuk DM, Lennon VA, Lucchinetti C, PitTock SJ, Weinshenker BG. The spectrum of neuromyelitis optica. Lancet Neurol 2007; 6: 805-815.

34. Volpe NJ. The Optic Neuritis Treatment Trial. A definitive answer and profound impact with unexpected results. Arch Ophthalmol 2008, 126: 996-999.

35. Calvetti O, Vignal-Clermont C, Drews-Botsch CD, Atkins EJ, Newman NJ, Biousse V. Prise en charge des névrites optiques isolées en France: enquete auprès des neurologues et des ophtalmologistes. Rev Neurol (Paris) 2008; 164: 233-241.

36. CoYle PK. Early treatment of multiple sclerosis to prevent neurologic damage. Neurology 2008; 71(24 Suppl 3): S3-7.

37. Jacobs LD, Beck RW, Simon JH, Brownscheidle CM, MurRey TJ, Simonian NA et al. Intramuscular interferon beta-1 treatment iniciated during a first demyelinating event in multiple sclerosis. CHAMPS Study group. N Engl J Med 2000; 343: 898-804.

38. CHAMPIONS Study Group. IM interferon beta-1 delays definite multiple sclerosis 5 years alter a first demyelinating event. Neurology 2006; 66: 678-684.

39. Comi G, Filippi M, Barkhof, Durelli L, Edan G, FERnÁNDEZ O et al. Early Treatment of Multiple Sclerosis Study Group. Effect of early interferon treatment on conversion to definite multiple sclerosis: a randomized study (ETOMS). Lancet 2001; 357: 1576-1582.

40. Kappos L, Polman CH, Freedman MS, Edan G, Hartung HP, Miller DH et al. Treatment with interferon beta-1b delays conversión to clinically definite and MCDonald MS in patients with clinically isolated síndromes. Neurology 2006; 67: 1242-1249. 
